\title{
Fabrication of Copper Nanowires from Aqueous Solution
}

\author{
Cindy Schmädicke ${ }^{1}$, Alexander Nerowski ${ }^{1}$, Larysa Baraban ${ }^{1}$, Lars David Renner ${ }^{1}$, Gianaurelio \\ Cuniberti, ${ }^{1,2}$ \\ ${ }^{1}$ Institute for Materials Science and Max Bergmann Center of Biomaterials, Dresden University of \\ Technology, 01062 Dresden, Germany, \\ g.cuniberti@tu-dresden.de \\ ${ }^{2}$ Division of IT Convergence Engineering, POSTECH, Pohang, Korea
}

\begin{abstract}
:
We report on the synthesis of copper nanowires from aqueous solution. The wires have an average diameter of up to $300 \mathrm{~nm}$ and a length of around $10 \mu \mathrm{m}$. A qualitative analysis of the growth parameters of copper nanowires was performed. We found that the morphology of the nanowires strongly depends on the concentration of the metal salt solution, the frequency of the applied AC voltage, and the peak-to-peak amplitude. The optimum parameters to fabricate straight and thin nanowires were analyzed. Resistance measurements at room temperature showed that the nanowires were conductive and showed ohmic behavior. The grown nanowires can be oxidized and readily used for gas sensing applications.
\end{abstract}

Key words: self-assembly, nanowires, copper, conductivity, ohmic behavior

\section{Introduction}

In this report, we present a single-step fabrication method of copper nanowires in solution by self-assembly. This technique will be relevant to sensor applications, because it has substantial advantages in comparison to other synthesis methods. It is cost-efficient, because only non-expensive equipment is used for production, the synthesis takes place within seconds, and the wires are already contacted, because they self-assemble between electrodes [1].

A number of diseases exhibit specific signatures or breath condensate markers, which can be detected in excess concentrations in the breath of diseased patients compared to healthy individuals, e.g., acetone in diabetes patients, NO in asthma patients [2]. In these cases a rapid and noninvasive breath analysis can be helpful. The development of such an analysis method is closely linked to recent technological progress. New technologies that ensure the fast analysis of breath can bring further advance for the diagnosis of disease. Nano-based gas sensors belong to those new trends and they can become valuable tools to provide widespread analysis devices, because of their high sensitivities, small sizes and low power requirements [3]. For these applications the controlled growth of very thin nanowires between electrodes is of particular interest. The grown nanowires are particularly suited for the detection of gas after nanowire oxidation, since they have a small diameter and due to their dendritic shape they have a large surface to volume ratio. A resistive gas sensor based on nanowires is illustrated schematically in Fig. 1.

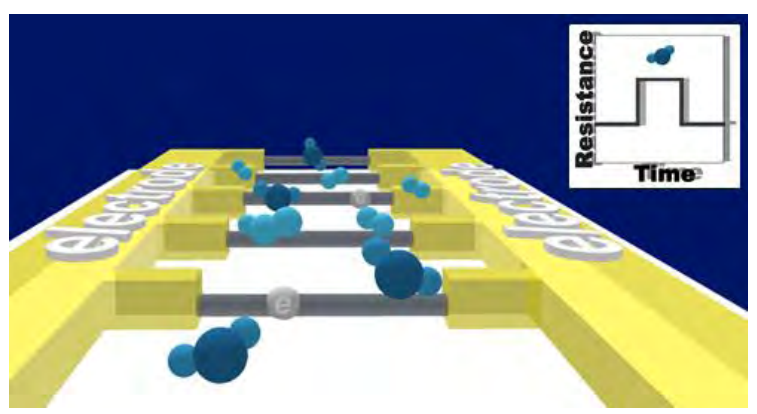

Fig. 1. Schematics of a nanowire based resistive gas sensor. The copper oxide nanowires grow simultaneously between the gold electrode contacts. The measurement signal is the resistance, which will change in dependence of the ambient gas concentration.

To study the nanowire growth process, we have controlled a series of process parameters, for example, the concentration of the solution, the frequency and the amplitude of the applied AC voltage.

\section{Materials and Methods}

Copper(II)-nitrate $\left(\mathrm{Cu}\left(\mathrm{NO}_{3}\right)_{2}\right) \quad$ (Sigma-Aldrich) was the basis for the metal salt solution. The stock solution was prepared by dissolving 0.1 , 0.5 , and $1 \mathrm{mM} \mathrm{Cu}\left(\mathrm{NO}_{3}\right)_{2}$ in deionized water. 
$1.5 \mu \mathrm{l}$ of the stock solution was then placed on the electrodes and an AC voltage with a specific form, frequency and amplitude was applied. As a result, the nanowires grew between the tips of the microfabricated gold electrodes on a glass substrate within seconds.

The electrodes were fabricated lithographically and the distance between them was fixed at $10 \mu \mathrm{m}$. Fig. 2 shows the schematic setup of two different electrode setups. The design of Fig. $2 b$ has an additional floating electrode (FE) which serves to reduce the Joule heating of the wire upon contact. This design is favorable because the magnitude of the current can be reduced by using floating electrodes [4]. The needles from a tip probe station (Karl Suss MicroTech) were placed in contact with the electrodes.

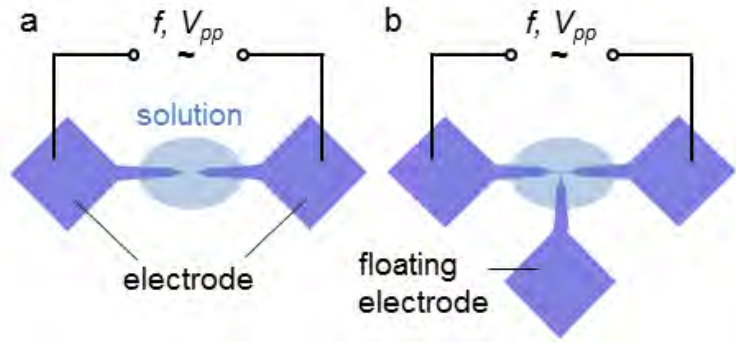

Fig. 2. Scheme of the experimental setup for nanowire growth with (a) two electrodes and (b) three electrodes.

A frequency generator (Tektronix AFG320) provided a rectangular voltage signal, which was observed with an oscilloscope (Tektronix TDS3014). The growth process was carried out at room temperature. The voltage was manually terminated at the moment the wires contacted the opposing electrode. For the resistance measurements a dual channel measurement instrument (Keithley $2602 \quad$ System SourceMeter) was used.

The produced nanowires were characterized by scanning electron microscopy (SEM, Philips XL 30 ESEM-FEG).

\section{Results and Discussion}

We fabricated copper nanowires from solution via self-assembly. The morphology of nanowires was investigated as a function of the metal concentration $c$, frequency $f$, and peak-topeak voltage $V_{p p}$.

We prepared copper nanowires from $\mathrm{Cu}\left(\mathrm{NO}_{3}\right)_{2}$ solutions with concentrations of $0.1,0.5$, and $1 \mathrm{mM}$ in deionized water. A peak-to-peak voltage of $6 \mathrm{~V}$ with a frequency of $100 \mathrm{kHz}$ was applied between the neighboring electrodes. In our experiments, we observed that by changing the concentration, we produced a variety of patterns of deposits (Fig. 3). It can be seen that increasing the copper concentration led to a higher wire density. This difference was probably caused due to an enhanced wire nucleation rate. On the other hand, we found that while decreasing the copper concentration the morphology of the grown nanowires changed to a more dendritic growth.
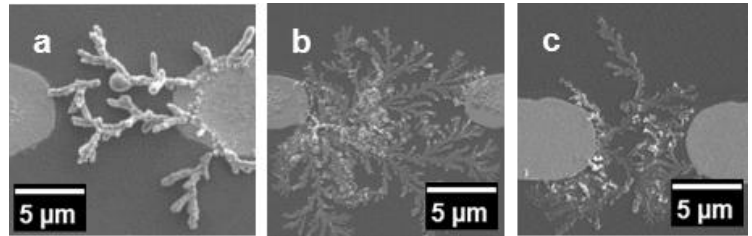

Fig. 3. Copper nanowires grown from copper salt solutions with concentrations of (a) $1 \mathrm{mM}$, (b) $0.5 \mathrm{mM}$, (c) $0.1 \mathrm{mM}$.

Frequency was found to be a further major parameter in controlling the process of nanowire growth. SEM images illustrate grown nanowires that were produced using $50 \mathrm{kHz}$, $250 \mathrm{kHz}, 500 \mathrm{kHz}$ and $750 \mathrm{kHz}$ frequencies, respectively (Fig. 4). A peak-to-peak voltage of $8 \mathrm{~V}$ was applied and the concentration of the solution was kept constant at $1 \mathrm{mM}$.
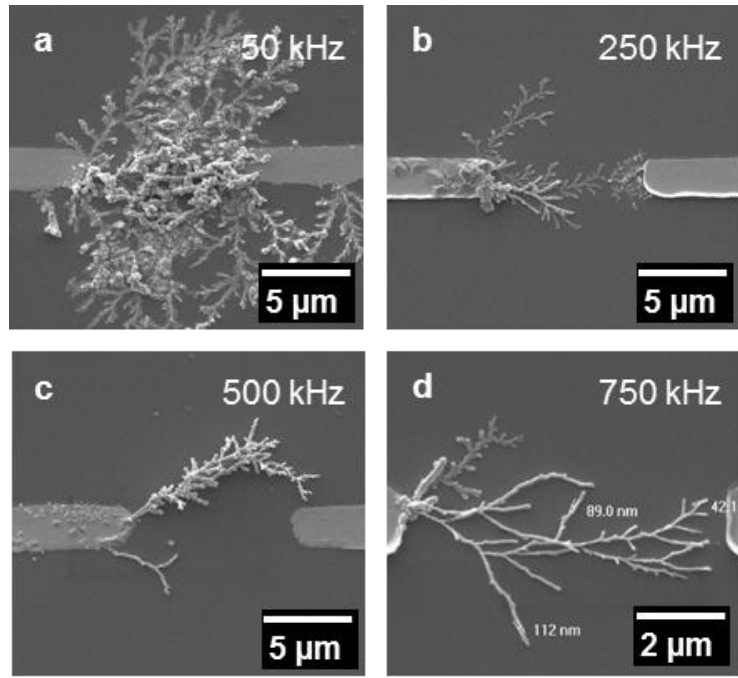

Fig. 4. Copper nanowires grown via self-assembly at different $A C$ field frequencies at a constant copper concentration of $1 \mathrm{mM}$ : (a) $50 \mathrm{kHz}$, (b) $250 \mathrm{kHz}$, (c) $500 \mathrm{kHz}$, (d) $750 \mathrm{kHz}$.

The grown wires have a diameter of $40-120 \mathrm{~nm}$ as determined from image analysis (for example Fig. 4d). Nanowires that were grown at low frequencies of values of 50 and $250 \mathrm{kHz}$ showed an extremely dendritic shape (Fig. 4 $a, b)$. The wires became straighter and thinner with increasing frequencies of 500 and $750 \mathrm{kHz}$ (Fig 4. c,d). Furthermore, we determined that an increase of the frequency led to a decrease in wire density.

In another experimental approach, we observed that the wire morphology could be manipulated by changing the magnitude of the applied 
voltage while keeping the other experimental parameters constant. Thus, the influence of the voltage on the wire growth was examined. Additionally, the electrode design of Fig. $2 b$ was used. As mentioned above, this layout lowered the Joule heating of the wire upon contact. This electrode design should prevent the wires from burning when they contacted the electrode. The influence of the floating electrode on the morphology has not been investigated in detail and will be examined in future works. The results are summarized in Fig. 5 showing the different morphology of the formed nanowires.
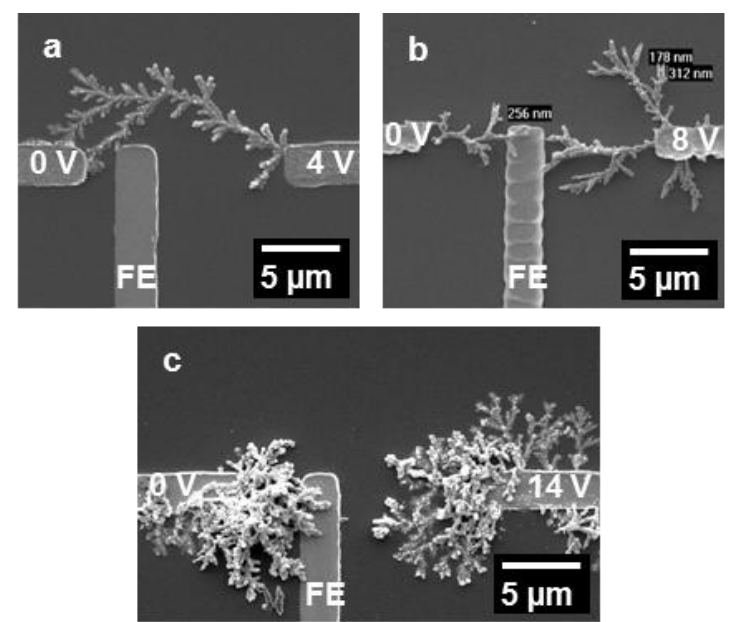

Fig. 5. SEM images of grown copper nanowires at constant $c=1 \mathrm{mM}$ and $f=50 \mathrm{kHz}$, but varying voltages (a) $V_{p p}=4 \mathrm{~V}$, (b) $V_{p p}=8 \mathrm{~V}$, (c) $V_{p p}=14 \mathrm{~V}$.

We used a constant copper solution concentration of $1 \mathrm{mM}$ and a frequency of $50 \mathrm{kHz}$ which resulted in branched wires that were about 100-300 nm thick. Analogous to the dependence on the salt concentration, we found that an increasing voltage resulted in a higher wire density. Moreover, we observed that with lower voltages the probability is increased to produce contacted nanowires, because lower voltages prevent nanowires from being immediately burned. The self-assembly process was reproducible over a wide experimental range.

In the following, we investigated the electrical properties of the wires. The resistances of these wires were measured at room temperature. The analysis of the resistances resulted in 150-450 $\Omega$ and the nanowires exhibited ohmic behavior (Fig. 6).

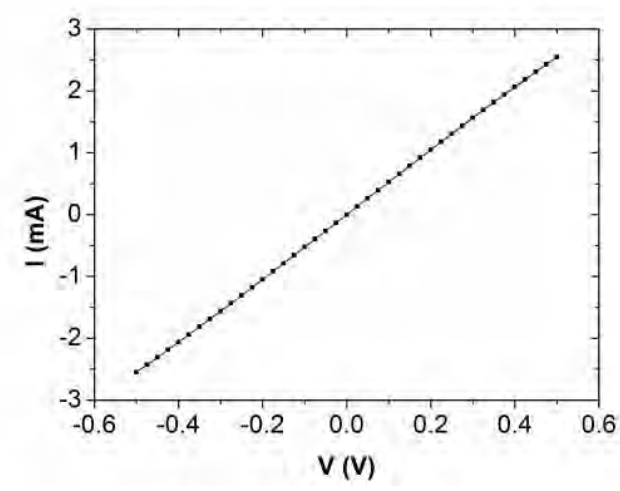

Fig. 6. The current-voltage (I-V) characteristics shows ohmic behavior of the nanowire. This was grown at $V_{p p}=8 \mathrm{~V}, f=100 \mathrm{kH}$, and $c=0.5 \mathrm{mM}$. The measured resistance was $188 \Omega$.

The resulting conductivity for a wire length of $10 \mu \mathrm{m}$ and a diameter of $300 \mathrm{~nm}$ was $752 \mathrm{kS} / \mathrm{m}$. This is approximately two orders of magnitude lower than the bulk conductivity of pure copper at room temperature. The result indicates that the nanowires possess a polycrystalline structure, crystallographic defects and a high contact resistance between nanowire and electrodes [5].

Fig. 7 gives an example of the resistance response of a dielectrophoretically fabricated copper nanowire. The graph illustrates the oxidation process of the nanowires in air. We interpolated this graph with an appropriate fit function. To quantify the oxidation process, we define the resistance change $(\Delta R)$ of our nanowire as

$\Delta R=1-\frac{R_{\text {start }}}{R_{\text {end }}}$

where $R_{\text {start }}$ and $R_{\text {end }}$ are the resistances at the beginning and at the end of the measurement interval, respectively. With this definition, it was found that for the exemplary experiment the resulted $\Delta R$ was approximately $2.5 \%$. We concluded that the small increment does suggest, the wire did not oxidize, because the resistance remains approximately constant over time. 


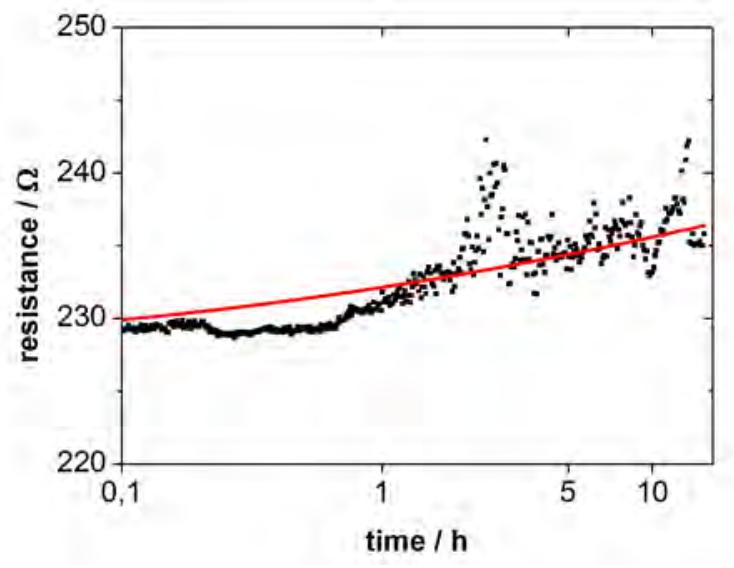

Fig. 7. Measurement of resistance over time for an exemplary copper nanowire.

\section{Conclusions}

In summary, we have demonstrated the selfassembly of one-dimensional copper nanowires. We fabricated micrometer sized nanowires from an aqueous copper salt solution. The length of the nanowires was defined by the gap between the electrodes. The operating parameters that influence the morphology of the nanowires were identified, and the effect of these parameters on the nanowire growth process was studied. To obtain straight and thin nanowires the applied electric field should be induced by a low voltage $(4 \mathrm{~V})$ with a high frequency $(750 \mathrm{kHz})$. The resistances of the copper nanowires were between 150-450 $\Omega$ and we observed perfect ohmic behavior. The grown nanowires can be easily and reproducibly synthesized with the advantages of having a large surface area to volume ratio as well as a low resistance. These properties provide the basis to produce nanowires of different metal salt solutions for gas sensing applications. By using parallel sets of electrode arrays, we have the ability to produce simultaneously multiple onedimensional nanowires. The described process is time-saving and allows the production of complex nanocircuits and thus nano-based sensors.

\section{Acknowledgments}

This work is funded by the European Union (ERDF) and the Free State of Saxony via the ESF project 100098212 InnoMedTec. We gratefully acknowledge support from the German Excellence Initiative via the Cluster of Excellence EXC 1056 "Center for Advancing Electronics Dresden" (cfAED). This research was supported by World Class University program funded by the Ministry of Education, Science and Technology through the National Research Foundation of Korea (R31-10100).

\section{References}

[1] A. Nerowski, M. Poetschke, M. Bobeth, J. Opitz, G. Cuniberti, Dielectrophoretic Growth of Platinum Nanowires: Concentration and Temperature Dependence of the Growth Velocity, Langmuir 28, 7498-7504 (2012); doi: 10.1021/la300302n

[2] A. Manolls, The Diagnostic Potential of Breath Analysis, Clinical Chemistry 29, 5-15 (1983)

[3] Y. Lu, C. Partridge, M. Meyyappan, J. Li, A carbon nanotube sensor array for sensitive gas discrimination using principal component analysis, J. Electroanal. Chem. 593, 105-110 (2006); doi: 10.1016/j.jelechem.2006.03.056

[4] S. Golan, D. Elata, M. Orenstein, U. Dinnar, Floating electrode dielectrophoresis, Electrophoresis 27, 4919-4926 (2006); doi: 10.1002/elps. 200600208

[5] C. Cheng, R. K. Gonela, Q. Gu, D. T. Haynie, Self-Assembly of Metallic Nanowires from Aqueous Solution, Nano Lett. 5, 175-178 (2005); doi: $10.1021 / \mathrm{nl} 048240 \mathrm{q}$ 